This document is the Accepted Manuscript version of a Published Work that appeared in final form in Analytical Chemistry, copyright (c) American Chemical Society after peer review and technical editing by the publisher. To access the final edited and published work see:

https://dx.doi.org/10.1021/acs. analchem.9b03898. 


\title{
Site-specific mRNA cleavage for selective and quantitative profil- ing of alternative splicing with label-free optical biosensors
}

\author{
Cesar S. Huertas, ${ }^{\mathrm{a}, \mathrm{b}, *}$ Sophie Bonnal, ${ }^{\mathrm{c}, \mathrm{d}}$ Maria Soler, ${ }^{\mathrm{e}, \mathrm{a}}$ Alfonso M. Escuela ${ }^{\mathrm{f}}$ Juan Valcárcel, ${ }^{\mathrm{c}, \mathrm{d}, \mathrm{g}}$ and Lau- \\ ra M. Lechuga ${ }^{a}$ \\ ${ }^{a}$ Nanobiosensors and Bioanalytical Applications Group, Catalan Institute of Nanoscience and Nanotechnology (ICN2), \\ CSIC, BIST and CIBER-BBN, 08193 Bellaterra, Barcelona, Spain \\ ' Integrated Photonics and Applications Centre, School of Engineering, RMIT University, Melbourne, VIC 3001, Australia \\ ${ }^{\mathrm{c}}$ Centre de Regulació Genòmica and BIST, 08003 Barcelona, Spain \\ ${ }^{d}$ Universitat Pompeu Fabra, 08003 Barcelona, Spain \\ ${ }^{\mathrm{e} N e t w o r k i n g ~ R e s e a r c h ~ C e n t e r ~ o n ~ B i o e n g i n e e r i n g, ~ B i o m a t e r i a l s ~ a n d ~ N a n o m e d i c i n e ~(C I B E R-B B N), ~ S p a i n ~}$ \\ fInstitute for Applied Microelectronics (IUMA). University of Las Palmas de Gran Canaria, E-35017 Las Palmas (Spain) \\ 'Institució Catalana de Recerca i Estudis Avançats, Spain
}

\begin{abstract}
Alternative Splicing of messenger RNA precursors is a key process in gene regulation, contributing to the diversity of proteomes by the alternative selection of exonic sequences. Most cancers are associated with alterations in this mechanism, enhancing their proliferation and survival, and can be employed as cancer biomarkers. Label-free optical biosensors are ideal tools for the highly sensitive and label-free analysis of nucleic acids. However, their application for alternative splicing analysis has been hampered due to the formation of complex and intricate long-range base-pairing interactions which make the direct detection in mRNA isoforms difficult. To solve this bottleneck, we introduce a new methodology for the generation of length-controlled RNA fragments from purified total RNA, which can be easily detected by the biosensor. The methodology seizes RNase H enzyme activity to degrade the upstream and downstream RNA segments flanking the target sequence upon hybridization to specific DNAoligos. It allows the fast and direct monitoring of Fas gene alternative splicing in real-time employing a Surface Plasmon Resonance (SPR) biosensor. We demonstrate the selective and specific detection of mRNA fragments in the pM-nM concentration range, reducing quantification errors and showing $81 \%$ accuracy when compared to RT-qPCR. The site-specific cleavage outperformed a random RNA hydrolysis by increasing the detection accuracy in $20 \%$, making this methodology particularly appropriate for label-free quantification of alternative splicing events in complex samples.
\end{abstract}

Gene regulation pathways play important roles in every process of life, including cell differentiation, metabolism, cell cycle, and signal transduction. There are many regulatory mechanisms that serve the cells to quickly adapt to environmental changes by modulating gene expression levels, establishing a complex and highly structured gene regulatory network. Alternative splicing (AS) is a key post-transcriptional process in the gene expression regulatory pathway. Splicing of precursor messenger RNA (Pre-mRNA) can generate different mature mRNAs that contain various combinations of exons, contributing to the diversity of proteomes. Alterations in AS processes can cause or modify the development of a variety of diseases including cancer ${ }^{1}$. Most cancers are associated with a switch in the splicing pattern of specific isoforms that provides them with proliferative capacity and survival properties ${ }^{2}$. In addition, splice variants diversify the repertoire of biomarkers and functionally contribute to drug resistance during chemotherapy $^{3}$. For example, Safikhani et al. demonstrated that isoform analysis represents a promising brand-new class of bi- omarkers for patient stratification in cytotoxic and targeted anticancer therapies ${ }^{4}$. Also, it has been demonstrated that RNA splicing events are crucial drivers of prostate cancer aggressiveness and therapeutic resistance in African American men ${ }^{5}$. Therefore, the evaluation of splice variants holds great promise for improving disease diagnosis and, ultimately, patient care in the era of precision medicine ${ }^{6}$. In fact, recent studies focusing on prostate cancer have demonstrated that mRNA analysis of circulating tumour cells could reveal substantial information on drug sensitivity and resistance ${ }^{7}$, which can lead to the development of less invasive approaches to modulate and monitor this process in therapeutic settings ${ }^{8,9}$.

The knowledge of the mechanisms and dynamics of AS has grown substantially in recent years, confirming its critical role in human health and disease. The gold-standard approach to characterize AS is reverse transcription of mRNA followed by polymerase chain reaction amplification (RT-qPCR) ${ }^{10}$. Also, microarrays have been designed to sample AS events on a genome scale and successfully used to examine AS across 
tissues, cellular states and species ${ }^{11,12}$. RNA-sequencing combined with computational tools have emerged as powerful tools for the examination of AS at a genome-wide level, providing opportunities to leverage AS to advanced diagnostics and prognostics tests for the delivery of effective medicines to patients. Recently, label-free optical biosensors have been pointed out as potential solutions for disease diagnostics and therapy follow-up by the analysis of nucleic acids ${ }^{13}$. These biosensors are high-sensitive analytical devices able to perform label-free assays in a timely and inexpensive manner, with minimal operator training and easy-to-perform procedures.

The Surface Plasmon Resonance (SPR) biosensor is considered the landmark in optical biosensors. SPR sensors have been widely employed in the pharmaceutical industry and research laboratories for the study of numerous types of biomolecular interactions ${ }^{14}$. SPR biosensors can detect, monitor, and quantify molecules attaching to the sensor surface by measuring the change in the refractive index (RI) produced at its immediate vicinity, thus avoiding amplification steps or molecular labelling. However, the most challenging factor in label-free detection of mRNAs is the target length. Long single-stranded RNA sequences are prone to generate secondary and tertiary structures through complex base-pairing and backbone interactions facilitated by the intrinsic flexibility of RNA moieties. These complex structures can hide the target sequence and hinder their accessibility to the bioreceptor monolayer. In addition, the production of nucleic acid sequences by conventional oligonucleotide synthesis processes has some limitation due to chemical reaction efficiency. As a result, the length of synthetic oligonucleotides typically do not exceed 150-200 nucleotides (nt $)^{15}$, which impedes the precalibration of the biosensor by employing standards for target sequences over $200 \mathrm{nt}$. This is one possible reason why there is a shortage of publications related to the detection of long RNA sequences with label-free optical biosensors ${ }^{13}$. Our team previously demonstrated the capabilities of an SPR biosensor for the label-free analysis of AS events ${ }^{16}$. In this study we showed that RNA fragmentation was necessary for the accurate assessment of AS events by the SPR biosensor, since the signal from these long-sequences was almost negligible probably due to the target sequences low accessibility. To do that, a sample pre-processing step was applied for the fragmentation of purified total RNA. The strategy followed takes advantage of the susceptibility of phosphodiester bonds from the ribose sugar phosphate backbone of RNA to be hydrolysed in alkaline medium ${ }^{17}$, generating $\sim 200$-nt mRNA fragments, which can be directly analysed by the SPR biosensor without PCR-amplification. However, the incubation time is a crucial parameter in this approach since it promotes a random cleavage and is prone to lose sample input by disruption of the sequence of interest. This time will also vary depending on the sample quality. Poor-quality samples could be caused by RNA degradation. Thus, additional alkaline degradation is not advisable because it can foster the loss of target input and bias the final biosensor readout. In such cases, it is important to keep RNA integrity to ensure an accurate analysis. Therefore, other methodologies should be considered in order to achieve a more controlled cleavage of RNA molecules.

Whereas DNA molecules can be easily cut at specific sequence sites by restriction enzymes, there are no specific tools for selective RNA sequence fragmentation. This disadvantage makes particularly difficult to generate RNA fragments of interest in a controlled manner. RNase $\mathrm{H}$ is an enzyme known to specifically degrade the RNA strand of RNA-DNA hybrid duplexes ${ }^{18}$. For example, it has been employed in the identification of a particular group of microbes based on the cleavage of a particular region in ribosomal RNAs (rRNAs) ${ }^{19}$. In this work, we introduce RNase H-mediated cleavage as a new methodology for the generation of specific RNA fragments of a length of interest and demonstrate its applicability for the real-time analysis of AS events using an SPR biosensor. The RNA site-specific cleavage employs two designed DNA oligonucleotides complementary to the upstream and downstream RNA segments flanking the specific RNA sequence of interest (Figure 1A). These DNA oligonucleotides, once hybridized to the endogenously transcribed AS isoforms, will promote the degradation of the hybridized RNA segment by RNase $\mathrm{H}$ activity, liberating the flanked sequence and making it available for its subsequent analysis by the SPR biosensor (Figure 1B). Compared to alkaline hydrolysis, which produces random RNA fragments, the RNase $\mathrm{H}$ method enables high precision fragmentation, thus ensuring that target RNA sequence is released intact with high selectivity and efficiency. Further, it offers the advantage of analysing the RNA fragments of interest directly in a PCR amplification-free mode and without risk of sample input loss, contrary to the alkaline hydrolysis approach.

The methodology is implemented for the evaluation of AS events in Fas gene mRNA isoforms, which play antagonistic roles in programmed cell death and are relevant to cancer. AS of Fas gene generates two isoforms depending on the inclusion or skipping of its exon 6 (ie. Fas567 or Fas57, respectively). Fas567 isoform encodes a full-length transmembrane protein receptor known as Fas/ CD95 that binds to Fas ligand (FasL), activating the signalling cascade of the extrinsic apoptotic pathway ${ }^{20}$. Fas 57 isoform lacks the trans-membrane domain, producing a soluble protein known as sFas that competes with the Fas receptor in the extra-cellular environment by binding to FasL. This version of Fas is overexpressed in cancer cells $^{21,22}$, and contributes to cancer aggressiveness ${ }^{23,24}$. We have optimized the RNase $\mathrm{H}$ cleavage conditions in terms of buffer composition, enzyme concentration and reaction temperature. Finally, the usefulness of the methodology has been demonstrated by the analysis of the endogenous expression of Fas gene alternative splicing isoforms in HeLa cells and its performance comparison with the gold-standard RTqPCR technique for validation. 
A

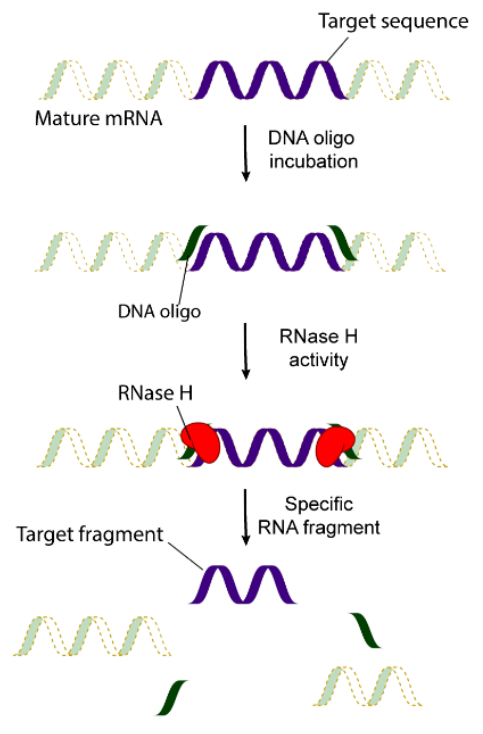

B

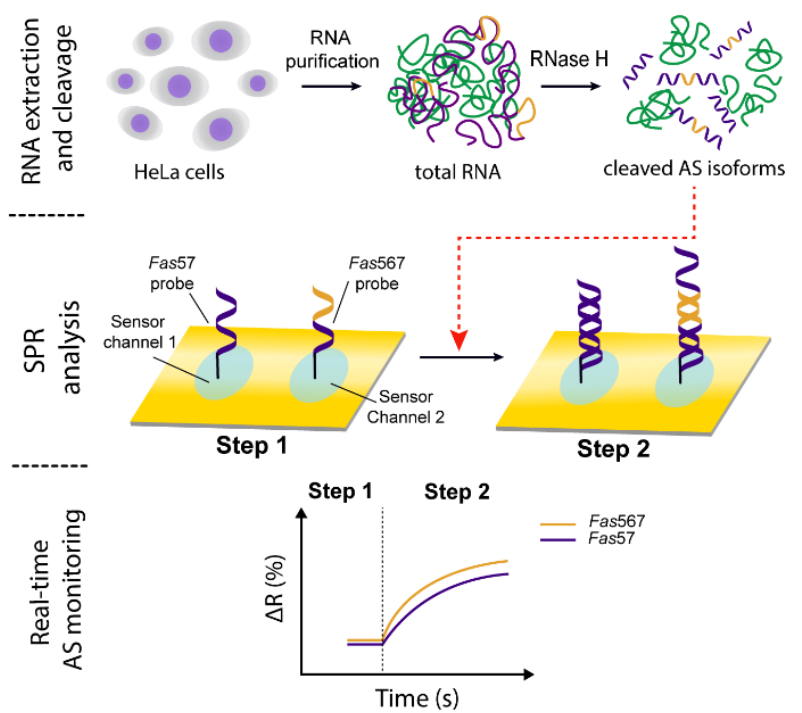

Figure 1. Scheme of the developed methodology. (A) RNase H specific cleavage process. (B) SPR biosensor methodology for specific detection of AS isoforms in HeLa cells.

\section{MATERIALS AND METHODS}

Chemical reagents and buffers. Solvents used for gold sensor chip cleaning were purchased to Panreac Applichem (Barcelona, Spain): Acetone $99.5 \%$ and Ethanol 99\%. Main salts and chemical reagents for sensor cleaning, buffer preparation and biofunctionalization were acquired from Sigma-Aldrich (Germany): Sodium Dodecyl Sulfate (SDS), Hydrochloric Acid ( $\mathrm{HCl}$ ), Sodium Phosphate monobasic (NaH2PO4) 99\%, Sodium Phosphate dibasic $\left(\mathrm{Na}_{2} \mathrm{HPO} 4\right) \geq 99 \%$, Sodium Chloride $(\mathrm{NaCl}) \geq 99.5 \%$, Sodium Citrate dihydrate $\geq 99 \%$, 6Mercapto-1-hexanol (MCH) $97 \%$ and Formamide $\geq 99.5 \%$.

Several buffers and solvents have been prepared either for functionalization or target analysis: PBS $50 \mathrm{mM}(50 \mathrm{mM}$ Phosphate buffer, $0.75 \mathrm{mM} \mathrm{NaCl}-\mathrm{pH} 7-)$, 20x SSC (3 M $\mathrm{NaCl}, 0.3 \mathrm{M}$ sodium citrate $-\mathrm{pH} 7-)$, 1x Alkaline hydrolysis buffer (Carbonate/Bicarbonate 50mM, EDTA 1mM, pH 9.2) and RNA fragmentation stop solution (3M Sodium Acetate), RNase $\mathrm{H}$ buffer $(50 \mathrm{mM}$ Tris- $\mathrm{HCl}, 75 \mathrm{mM} \mathrm{KCl}, 3 \mathrm{mM}$ $\mathrm{MgCl} 2,10 \mathrm{mM}$ DTT -pH 8.3-). Buffer solutions were prepared by using $\mathrm{H} 2 \mathrm{O}$ milliQ incubated $\mathrm{O} / \mathrm{N}$ with $2 \%$ DEPC and autoclaved at $121^{\circ} \mathrm{C}$ for 1 hour.

DNA sequences. All DNA-probes and synthetic targets were purchased from IBA GmbH (Goettingen, Germany) and are summarized in Table 1. DNA-probe sequences incorporate a thiol group at the 5'-end to enable chemical coupling with the gold sensor surface. In addition, a spacing region, which consists of a 15-thymine sequence (poly $\mathrm{T}_{15}$ ) is placed between the thiol group and the matching region. This sequence will not participate in the hybridization event but, instead, it acts as a vertical spacer due to the low affinity of thymine bases for gold surfaces ${ }^{25}$. The length of this poly $\mathrm{T}_{15}$ has been demonstrated to outperform the biosensor performance compared to other lengths ${ }^{26}$. mRNA isoform sequences were obtained from Ensembl genenome browser (http://www.ensembl.org/index.html). DNA/RNA sequences employed are summarized in Table 1.
SPR biosensor. The SPR biosensor device is based on Kretschmann configuration and monitors refractive index (RI) changes in real time ${ }^{27}$. A schematic illustration of the SPR biosensor is shown in the Suplementary Material (Figure S1). A p-polarized light of $670 \mathrm{~nm}$ from a laser source is divided in two identical beams focused on the crystal-backside of the gold sensor chip (glass surface coated with $2 \mathrm{~nm}$ of chromium and $45 \mathrm{~nm}$ of gold, $10 \times 10 \times 0.3 \mathrm{~mm} 3$, SSens, Enschede, The Netherlands). Measurements are performed at a fixed angle of incidence. Variations of the RI are detected due to the biointeraction events occurring at the sensor surface as changes in the reflected light intensity by a multielement photodiode. The flow system consists of two flow cells (300 nL each) for independent analysis. The device incorporates all optics, electronics and fluidics components necessary to operate autonomously. Sensograms reproduce the binding event by monitoring the increase (or decrease in case of unbinding events) of the intensity of the reflected light ( $\Delta$ Reflectivity $(\%), \Delta \mathrm{R}(\%))$ vs. time (seconds, s). This change of the intensity of the reflected light is directly related to changes in the RI of the dielectric medium caused by mass changes on the metallic sensor surface.

DNA-probe immobilization. Prior to DNA-probe immobilization, gold sensor chips were cleaned by consecutive sonication cycles $(1 \mathrm{~min})$ with solvents of increasing polarity (i.e. acetone, ethanol and $\mathrm{dH}_{2} \mathrm{O}$ ) previously heated up to their boiling point. Then, substrates were dried under nitrogen flux and placed in a $\mathrm{UV} / \mathrm{O}_{3}$ generator (BioForce Nanosciences, USA) for $20 \mathrm{~min}$. After that, gold sensor chips were subsequently rinsed with ethanol and water and dried under nitrogen flux. The gold sensor chip was then placed into the SPR device. 
Table 1. Sequences and probes and DNA/RNA synthetic targets employed.

\begin{tabular}{|c|c|}
\hline Name & Sequence \\
\hline Fas57 probe & 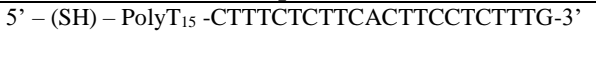 \\
\hline Fas56 probe & $\begin{array}{l}\text { 5'-(SH)- } \text { PolyT }_{15-} \text { AGATCTGGATCCTTCCTCTTTG-3' } \\
\text { 5'ATGTAGAACATGGAATCATCAAGGAATGCACAC }\end{array}$ \\
\hline $\begin{array}{l}\text { Fas57 } \\
\text { isoform }\end{array}$ & $\begin{array}{l}\text { TCACCAGCAACACCAAGTGCAAAGAGGAAGTGAA } \\
\text { GAGAAAGGAAGTACAGAAAACATGCAGAAAGCAC } \\
\text { AGAAAGGAAAACCAAGGTTCTCATGAATCTCCAA } \\
\text { CTTTAAATCCT3' } \\
\text { 5'ATGTGAACATGGAATCATCAAGGAATGCACACT } \\
\text { CACCAGCAACACCAAGTGCAAAGAGGAAGGATCC }\end{array}$ \\
\hline $\begin{array}{l}\text { Fas567 } \\
\text { isoform }\end{array}$ & $\begin{array}{l}\text { AGATCTAACTTGGGGTGGCTTTGTCTTCTTCTTTTG } \\
\text { CCAATTCCACTAATTGTTTGGGTGAAGAGAAAGGA } \\
\text { AGTACAGAAAACATGCAGAAAGCACAGAAAGGAA } \\
\text { AACCAAGGTTCTCATGAATCTCCAACTTTAAATCC } \\
\text { T3' }\end{array}$ \\
\hline DNA $_{1}$ probe & 5'-(SH)-PolyT15-ATCACCGACAGCGT3' \\
\hline $\mathbf{R N A}_{1}$ & 5'AACAUUCAACGCUGUCGGUGAGU3' \\
\hline Control & $\begin{array}{l}\text { 5'GAGGCAGGCGACGAGTTTGAACTGCGGTACCGG } \\
\text { CGGGCATTCAGTGACCTGACATCCCAGCTCCACAT } \\
\text { CACCCCAGGGACAGCATATCAGAGCTTTGAACAG } \\
\text { GATACTTTTGTGGAACTCTATGGGAACAATGCAGC } \\
\text { AGCCGAGAGCCGAAAGGGCCAGGAACGCTTCAAC } \\
\text { CGCTGGTTCCTGACGGGCATGACTGTGGC3' }\end{array}$ \\
\hline
\end{tabular}

For RNase $\mathrm{H}$ activity optimization, formation of a selfassembled monolayer (SAM) of $\mathrm{DNA}_{1}$ probe was carried out in-situ on the gold sensor chip by flowing $250 \mathrm{~mL}$ of a solution containing $1 \mu \mathrm{M}$ of $\mathrm{DNA}_{1}$ probe in $1 \mathrm{x}$ PB buffer solution at a $12 \mathrm{~mL} / \mathrm{min}$ rate. For Fas gene isoform detection, formation of mixed SAMs of either Fas56-probe or Fas57probe/MCH $(20: 1,1 \mu \mathrm{M})$ was carried out in-situ on the gold sensor chip by flowing $250 \mathrm{~mL}$ of the mix in 1x PB buffer solution at a $12 \mathrm{~mL} / \mathrm{min}$ rate. We immobilized each isoform probe in independent SPR channels.

Alkaline hydrolysis of purified total RNA from HeLa cells. Total RNA from human cervical carcinoma HeLa cells (approx. 1.5 million cells) was isolated using the RNeasy kit (Qiagen, Germany). For alkaline hydrolysis, samples were preheated at $95^{\circ} \mathrm{C}$ for $15 \mathrm{~s}$ and mixed with preheated 1xAlkaline hydrolysis buffer (Carbonate/Bicarbonate $50 \mathrm{mM}$, EDTA $1 \mathrm{mM}, \mathrm{pH} 9.2)$ to start the hydrolysis reaction. Total RNA samples were exposed to the alkaline buffer for 3 minutes. After each incubation time, samples were placed on ice and reaction was stopped with RNA fragmentation stop solution (3M Sodium Acetate, $\mathrm{pH}$ 5.2). After applying the fragmentation protocol, endogenous levels of Fas isoforms were checked using the SPR biosensor.

RNase $H$ cleavage of purified total RNA from HeLa cells. Total RNA from human cervical carcinoma HeLa cells (aprox. 1.5 million cells) was isolated using the RNeasy kit (Qiagen, Germany). They were transfected at $50 \%$ confluency with 0.4 micrograms of Fas minigenes ${ }^{28}$ and 10 micrograms of plasmid DNA expressing either beta galactosidase or PTB, per culture plate of $10 \mathrm{~cm}$. When 6-wells plates were used, $50 \mathrm{ng}$ minigene and 3 micrograms of expression plasmid DNA were transfected per well. When minigene and protein expression vectors were used, cells were collected 48 hours posttransfection. Total RNA was isolated using the RNeasy kit (Qiagen) and the pattern of alternative splicing was analyzed by RT-PCR. Reverse transcription was carried out using AMV-Reverse Transcriptase (Promega) and specific reverse primers $^{28}$. Polymerase chain reaction was carried out using GoTaq (Promega) and forward and reverse primers corre-

sponding to the exonic regions flanking the alternatively spliced sequences ${ }^{29}$. For SPR biosensor analysis, two DNAoligos were designed, complementary to the sequences flanking exon 5 ( 3 ' end of exon 4) and exon 7 ( 5 ' end of exon 8) (see Table 2). These sequences, once hybridized to the endogenously transcribed isoforms, will promote the degradation of the RNA sequence in contact, producing fragments of approximately 200 nts (Table 2). We incubated total RNA with 10 $\mu \mathrm{M}$ concentration of each DNA-probe at $95^{\circ} \mathrm{C}$ for $2 \mathrm{~min}$ and cooled it on ice for $5 \mathrm{~min}$ for probe-annealing. After annealing, RNA samples were incubated with $100 \mathrm{U} / \mathrm{mL}$ of RNase $\mathrm{H}$ in $1 \mathrm{x}$ RNase $\mathrm{H}$ buffer $(50 \mathrm{mM}$ Tris- $\mathrm{HCl}, 75 \mathrm{mM} \mathrm{KCl}, 3 \mathrm{mM}$ $\mathrm{MgCl}$, $10 \mathrm{mM}$ DTT - $\mathrm{pH}$ 8.3-) at room temperature (RT) for $60 \mathrm{~min}$. RNase $\mathrm{H}$ was later inactivated by incubating at $65^{\circ} \mathrm{C}$ for $15 \mathrm{~min}$. After applying the fragmentation protocol, endogenous levels of Fas isoforms were evaluated using the SPR biosensor.

Isoform hybridization and biosensor regeneration. Splicing variants detection was performed by injection of the target splicing variant samples into the biosensors at a $15 \mu \mathrm{L} / \mathrm{min}$ rate and subsequent hybridization with their complementary DNA-probes immobilized on the sensor surface. These samples were dissolved in the hybridization buffer $(3 \mathrm{xSSC}-0.45$ $\mathrm{M}$ in $\mathrm{NaCl}, 0.045 \mathrm{M}$ sodium citrate- with a $45 \%$ formamide). Isoform-probe interactions were disrupted by using a $50 \%$ formamide in aqueous solution. Calibration curves were obtained for each DNA probe by triplicate measurements of the DNA Fas gene AS isoforms synthetic standards at different concentrations $(2.5,5,10,20,30$, and $50 \mathrm{nM})$. The mean and the standard deviation (SD) of each concentration were plotted versus the target concentration and fitted to a curve. Fragmented and non-fragmented total RNA samples from HeLa cells were diluted in hybridization buffer to $20 \mathrm{ng} / \mu \mathrm{L}$ and analysed by SPR biosensor at the same conditions used for the standards. In addition, purified total RNA from bacteria (XLI Blue) was used as a negative control.

Table 2. DNA probes for RNase $\mathrm{H}$ cleavage and Fas mRNA fragments generated after the cleavage.

\begin{tabular}{|c|c|c|}
\hline Name & Length (nt) & Sequence \\
\hline $\begin{array}{l}\text { Fas } 567 \\
\text { isoform } \\
\text { fragment } \\
\text { after } \\
\text { RNase H }\end{array}$ & 214 & $\begin{array}{l}\text { 'CCAAAUGUGAACAUGGAAUCAUCAAGG } \\
\text { AAUGCACACUCACCAGCAACACCAAGUG } \\
\text { CAAAGAGGAAGGAUCCAGAUCUAACUUG } \\
\text { GGGUGGCUUUGUCUUCUUCUUUUGCCAA } \\
\text { UUCCACUAAUUGUUUGGGUGAAGAGAAA } \\
\text { GGAAGUACAGAAAACAUGCAGAAAGCAC } \\
\text { AGAAAGGAAAACCAAGGUUCUCAUGAAU } \\
\text { CUCCAACUUUAAAUCCU3' }\end{array}$ \\
\hline $\begin{array}{l}\text { Fas } 57 \\
\text { isoform } \\
\text { fragment } \\
\text { after } \\
\text { RNase H }\end{array}$ & 149 & $\begin{array}{l}\text { 5'CCAAATGTGAACATGGAATCATCAAGG } \\
\text { AATGCACACTCACCAGCAACACCAAGTGC } \\
\text { AAAGAGGAAGTGAAGAGAAAGGAAGTAC } \\
\text { AGAAAACATGCAGAAAGCACAGAAAGGA } \\
\text { AAACCAAGGTTCTCATGAATCTCCAACTT } \\
\text { TAAATCCT3' }\end{array}$ \\
\hline $\begin{array}{l}\text { Fas exon } \\
4 \text { probe }\end{array}$ & 25 & 5' TGCAAGGGTCACAGTGTTCACATAC 3' \\
\hline $\begin{array}{l}\text { Fas exon } \\
8 \text { probe }\end{array}$ & 25 & 5' CAGATAAATTTATTGCCACTGTTTC 3' \\
\hline
\end{tabular}



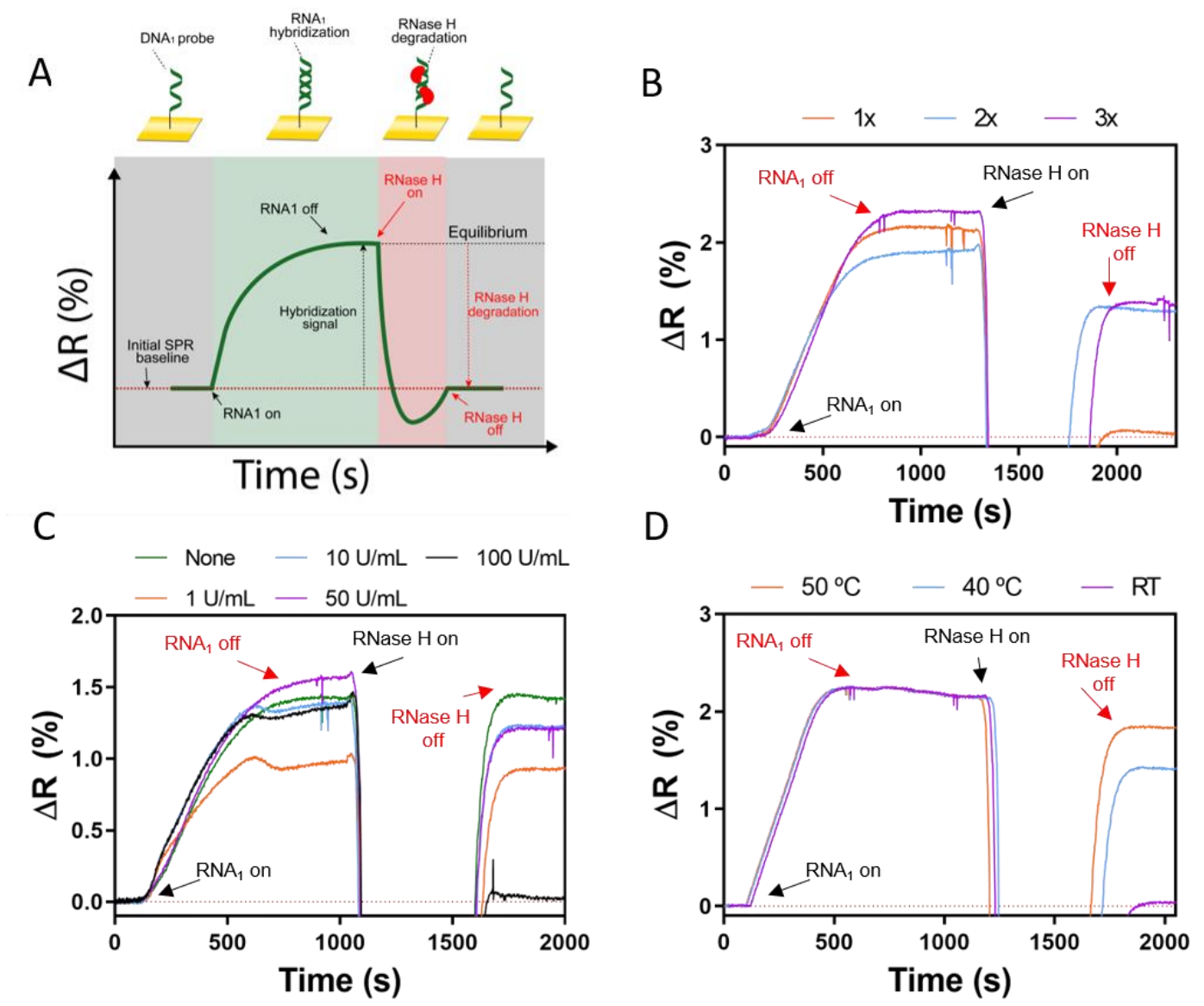

Figure 2. Optimization of RNase H conditions for Fas gene alternative splicing analysis through SPR biosensor. (A) Scheme of the strategy followed for the optimization of the RNase H activity by SPR analysis. (B) RNase H buffer concentration optimization. (C) RNase H concentration optimization. (D) Temperature optimization for RNase H activity optimization (RT: Room Temperature).

Data analysis. The data were collected and analysed using Origin 8.0 software (OriginLab, Northampton, MA). Graphical representation and calibration curve interpolation was performed in Graphpad prism 8 (Graphpad Software, San Diego, CA -EEUU-) The experimental detection limit (LOD) was defined as the target concentration providing a $\Delta \mathrm{R}(\%)$ in the hybridization signal at least three times higher than that of the standard deviation of the control signal. The coefficients of variation were obtained as the ratio of the standard deviation to the mean, expressed in percentages $(\% \mathrm{CV})$. Agreement between RT-qPCR and SPR was assessed by applying the Bland-Altman model for replicate measurements.

\section{RESULTS AND DISCUSSION}

\section{Specific RNA cleavage optimization.}

The assessment of the RNase $\mathrm{H}$ activity was carried out using a portable custom-designed SPR biosensor ${ }^{27}$. This SPR biosensor is based on Kretschmann configuration and monitors refractive index changes at a fixed angle of incidence. It enables analysis in real time, offering the advantage of monitoring biological interactions as they happen.

The biosensor response is represented in sensograms that reproduce the binding event by monitoring the increase (binding events) or decrease (unbinding events) of the intensity of the reflected light $(\Delta$ Reflectivity $(\%), \Delta \mathrm{R}(\%))$ vs. time (seconds, $\mathrm{s})$. This change of the intensity of the reflected light is directly related to the mass changes on the gold surface, which correspond to the concentration of the hybridized RNA. The light source is divided into two identical beams, generating a surface plasmon in two different flow-cells of $300 \mathrm{~nL}$ each. This allows for parallel, independent analyses of two different samples. We took advantage of this process to assess and optimize RNase $\mathrm{H}$ activity exploring different parameters of the reaction, including: (i) the reaction buffer concentration, (ii) the enzyme concentration, and (iii) the reaction temperature.

We employed a short RNA sequence $\left(\mathrm{RNA}_{1}\right)$ (Table 1$)$ for the evaluation of the activity of the enzyme. We immobilized a specific complementary DNA probe $\left(\mathrm{DNA}_{1}\right.$ probe Table 1$)$ for the specific capture of $\mathrm{RNA}_{1}$ and, subsequently, monitored the RNA hybridization and RNase $\mathrm{H}$ activity in real time (Figure 2A). A change in the refractive index will be observed during the specific hybridization of the complementary $\mathrm{RNA}_{1}$ target, which will be directly related to its concentration in the analysed sample. Then, the interaction will find its equilibrium state and will reach a plateau in the SPR sensor signal. The net change in the baseline, expressed as variation of reflectivity $(\Delta \mathrm{R}(\%))$, will be directly proportional to the concentration of the hybridized target sequence. RNase $\mathrm{H}$ can be employed during the examination of RNA sequences in SPR biosensors for the regeneration of the sensor surface. The regeneration is underpinned by the RNase $\mathrm{H}$-induced degradation of the RNA sequences previously hybridized to the immobilized DNA 
probes, rendering them available for further analyse ${ }^{30}$. Thus, eventually, the hybridization will be interrupted by the RNase $\mathrm{H}$ solution, degrading $\mathrm{RNA}_{1}$ sequence, and returning the baseline to its original level.

First, we evaluated effect of the reaction buffer on RNase $\mathrm{H}$ activity. The buffer composition, $\mathrm{pH}$, and ionic strength of the solution are known to influence enzymatic cleavage efficiency. The optimum buffer composition for RNase $\mathrm{H}$ activity has been established and reported elsewhere (i.e., $50 \mathrm{mM}$ Tris$\mathrm{HCl}, 75 \mathrm{mM} \mathrm{KCl}, 3 \mathrm{mM} \mathrm{MgCl}$, $10 \mathrm{mM}$ DTT, pH 8.3) ${ }^{19}$. However, the concentration of salts must be tested for specific applications. The study was carried out as depicted in Figure 2A.

A DNA $_{1}$ probe was immobilized in-situ on the gold sensor chip and continuous $5 x$ saline-sodium citrate (5x SSC) buffer at $\mathrm{pH} 7$ was supplied over the functionalized sensor surface until a baseline was reached. Then, $\mathrm{RNA}_{1}$ was hybridized at a $50 \mathrm{nM}$ concentration in 5x SSC buffer with its specific receptor attached to the biosensor surface. Once we reached the equilibrium of the interaction, we flowed $250 \mathrm{U} / \mathrm{mL}$ of the enzyme at different RNase $\mathrm{H}$ buffer concentrations (1x, 2x and $3 \mathrm{x})$ at RT (i.e. $25^{\circ} \mathrm{C}$ ). The capability of the enzyme at each buffer concentration to return the sensor signal to the initial level due to the cleavage was evaluated. As can be observed in Figure 2B, the SPR signal dropped drastically below the initial baseline during the RNase $\mathrm{H}$ activity (RNase on). This dip in the signal was mainly caused by the difference in the RNase $\mathrm{H}$ buffer composition compared to the continuous buffer, producing a drastic drop in the refractive index of the medium and obscuring the monitoring of the degradation process. After RNase $\mathrm{H}$ treatment, the sensor surface was washed again with the running buffer and the net change between the sensor signal at the equilibrium of interaction and the new baseline provided the approximate \% of degraded RNA by the enzyme's activity. At a $2 x$ buffer concentration, the baseline did not return to its initial level, indicating that only a $48 \%$ of the hybridized RNA was degraded. A similar result was observed at $3 x$ buffer concentration. On the contrary, the baseline completely returned to the initial level at a $1 \mathrm{x}$ buffer concentration, confirming highest RNase $\mathrm{H}$ activity at this buffer concentration.

Once $1 \mathrm{x}$ was established as the proper buffer concentration, we tested the optimal enzyme concentration needed for the reaction. We injected four different concentrations of RNase $\mathrm{H}$ enzyme: none, 1, 10, 50 and $100 \mathrm{U} / \mathrm{mL}$. As depicted in Figure $2 \mathrm{C}$, the latter cleaved the hybridized RNA almost completely and achieved the same results as when using $250 \mathrm{U} / \mathrm{mL}$ while lower concentration failed in the full degradation of the RNA, indicating that a concentration of $100 \mathrm{U} / \mathrm{mL}$ of RNase $\mathrm{H}$ was sufficient to carry out the complete cleavage of the sequence. Finally, we explored the enzyme activity at different reaction temperatures. We tested room temperature (RT), $40{ }^{\circ} \mathrm{C}$ and 50 ${ }^{\circ} \mathrm{C}$ by preheating the RNase $\mathrm{H}$ solution before flowing it in the SPR biosensor. At $40{ }^{\circ} \mathrm{C}$ and $50{ }^{\circ} \mathrm{C}$, the enzyme was not fully effective, cleaving less than half of the hybridized RNA (Figure 2D). On the contrary, RT conditions achieved the best results, returning the sensor signal to the initial levels. According to these results, we considered that optimal conditions for RNase $\mathrm{H}$ activity were $100 \mathrm{U} / \mathrm{mL}$ enzyme concentration diluted in a $1 \mathrm{x}$ reaction buffer at $\mathrm{RT}$.
Comparative study of mRNA fragmentation procedures for Fas gene AS evaluation.

The optimized procedure for specific mRNA fragmentation by the RNase H enzyme was applied and evaluated for selective biosensing of endogenous levels of Fas gene AS isoforms directly from HeLa cells samples. We also compared its performance with non-fragmented and with alkaline-hydrolyzed samples.

RNase H cleavage was performed off-sensor on purified RNA from HeLa cells to assess the expression levels of Fas gene mRNA isoforms by following the experimental procedure depicted in Figure 1. In order to obtain Fas gene isoform fragments of interest, we designed 25 nt DNA sequences complementary to the sequences flanking exon 5 (3' end of exon 4 ) and exon 7 ( 5 ' end of exon 8 ). These sequences, once hybridized to the endogenously transcribed isoforms, should promote the degradation of the hybridized RNA sequence, producing fragments of approximately $200 \mathrm{nt}$ (Table 2). For comparison, alkaline hydrolysis was performed in parallel as previously described ${ }^{16}$ : RNA samples were preheated at $95{ }^{\circ} \mathrm{C}$ for $15 \mathrm{~s}$, mixed them with preheated $1 \mathrm{x}$ alkaline hydrolysis buffer and incubated the mix for 3.5 min (incubation time was previously optimized in order to obtain RNA fragments of an average of $200 \mathrm{nt}$ ) (Figure S2).

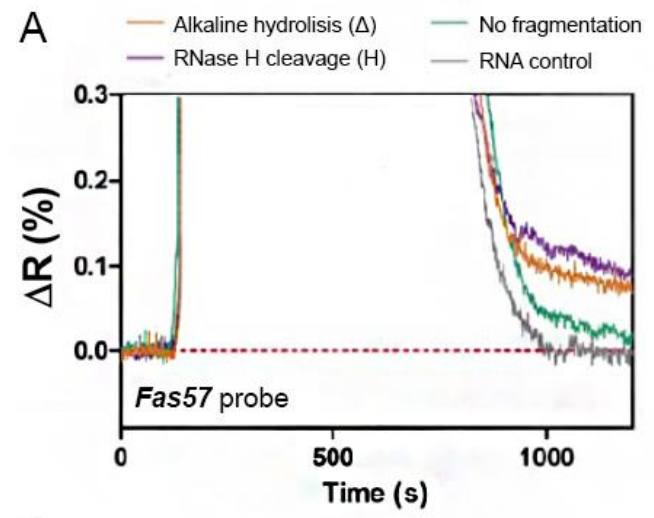

B

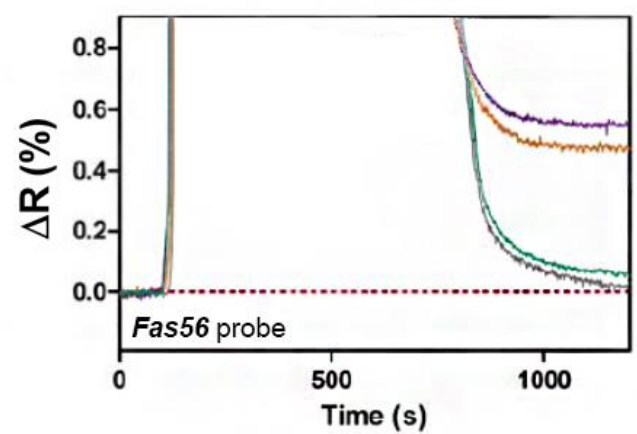

Figure 3. SPR sensograms of the detection of $20 \mathrm{ng} / \mu \mathrm{L}$ of total HeLa cell RNA for the endogenous expression of both (A) Fas567 isoform and (B) Fas57 isoform after applying RNase H cleavage $(\mathrm{H})$ (purple line), the alkaline hydrolysis $(\Delta)$ (orange line) and without any sample fragmentation (0) (green line). The grey line represents the signal given by the RNA control sample. Adapted from ${ }^{16}$ with permission of Elsevier.

To stop RNA degradation, samples were placed on ice and mixed with sodium acetate ( $\mathrm{pH}$ 5.2). The time of incubation is crucial and will strongly depend on the concentration and 
quality of the samples. Lower RNA concentrations will be degraded faster, leading to shorter fragments for similar incubation times. Likewise, poor-quality samples will already present some level of degradation and could lead to sample input losses. Therefore, it should be carefully optimized in each case.

After each fragmentation process, samples were diluted in the hybridization buffer to $20 \mathrm{ng} / \mu \mathrm{L}$ of purified total RNA. In order to detect Fas gene AS isoforms through SPR, a clean gold sensor chip was activated and placed into the SPR device. Immobilization of Fas 57 and Fas 56 probes was carried out in independent microfluidic cells. Mixed SAMs of either Fas 567 or Fas57 probes and alkanethiol 6-mercapto-1-hexanol (MCH) at a ratio of 20:1 were prepared and flowed over two different SPR sensor channels. MCH is a commonly used backfiller that acts as a lateral spacer, improving the target accessibility by minimizing steric hindrance forces created between the DNA probes in densely packed monolayers. After that, the mRNA samples obtained from HeLa cells after fragmentation are flowed over the functionalized sensor surface, and the specific Fas57 and Fas567 are captured and detected in real time. We monitored the SPR response recorded for each isoform in both SPR sensor channels. Results obtained using RNase H cleavage $\left(\mathrm{SPR}^{\mathrm{H}}\right)$ and alkaline hydrolysis protocol $\left(\mathrm{SPR}^{\Delta}\right)$ were compared with non-fragmented samples $\left(\mathrm{SPR}^{0}\right)$. Figures $3 \mathrm{~A}$ and $B$ show the SPR responses obtained after injection of HeLa cell total RNA samples for each receptor. SPR sensograms showed a significant increment of the sensor signal after sample injection time $(\approx 100$ s) due to differences in the refractive index of the hybridization buffer. After sample interaction, we washed the sensor surface with the continuous running buffer and analyzed the net changes in the baseline levels, which will correspond to the quantity of target hybridized to the biosensor. As can be seen in Figure 3, endogenous RNAs without any cleavage generated SPR responses of $0.02 \%$ and $0.09 \% \Delta \mathrm{R}$ for Fas 57 and Fas 567 , respectively. In contrast, samples treated with RNase $\mathrm{H}$ produced an $\Delta \mathrm{R}$ of $0.12 \%$ and $0.57 \%$ for Fas 57 and Fas 567 , respectively. Alkaline hydrolysed samples showed increments in the SPR reflectivity of $0.09 \%$ (Fas57) and $0.49 \%$ (Fas567). Negative control RNA purified from bacteria produced negligible SPR sensor responses, returning to the baseline level and confirming the specificity of the detection. Therefore, the application of either of the fragmentation protocols resulted in an increment in the SPR sensor responses for both isoforms. These results confirm our hypothesis that RNA fragmentation increase the accessibility of the target to the monolayer and, therefore, enhance the SPR detection response.

Validation of the measurement results using RT-qPCR.

In order to obtain quantitative data of Fas AS isoform levels, we carried out calibration curves using synthetic DNA standard sequences (Fas567 and Fas57 isoforms, Table 1) corresponding to the exonic regions 567 and 57 of Fas gene AS isoforms (Figures 4A and 4B). These DNA sequences were designed with lengths of 150-200 nt to simulate the fragment lengths obtained by the fragmentation processes.
A

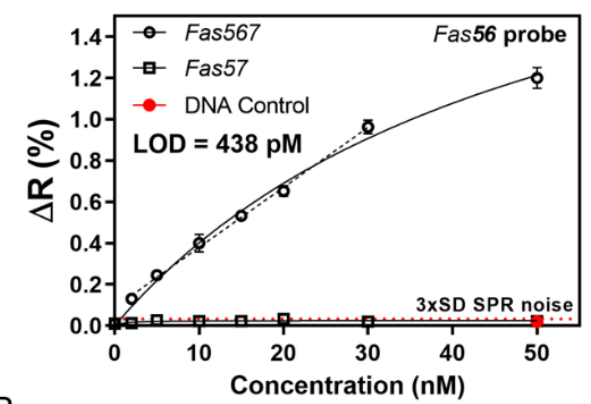

B

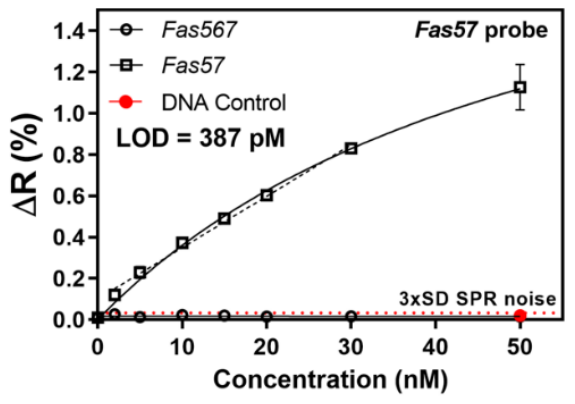

C

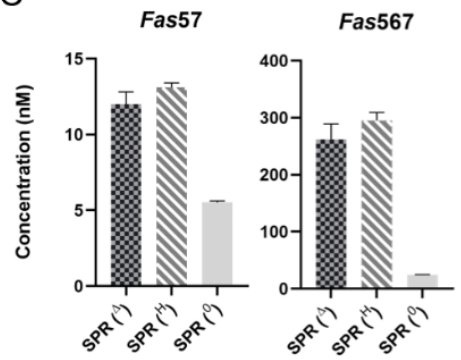

D

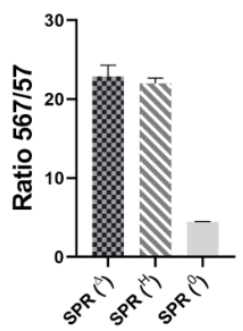

Figure 4. Quantitative analysis of mRNA Fas gene isoforms by SPR. SPR calibration plots for Fas57 (A) $(\mathrm{R} 2=0.99)$ and Fas56 (B) $(\mathrm{R} 2=0.99)$ probes using the optimized hybridization and detection conditions. Solid lines correspond to the non-linear fit of the calibration curves. Red dashed line corresponds to 3xSD SPR noise, which is the limit for the minimum signal detectable. All data show mean \pm SD of triplicate measurements. (C) Histograms comparing the analytical quantification of Fas57 (left) and Fas567 (right) isoforms. (D) Histogram comparing the 567/57 ratio after each condition tested by SPR. All data show mean \pm $\mathrm{SD}$ of duplicate measurements. Figure A and B are reprinted from ${ }^{16}$ with permission of Elsevier.

We analysed different concentrations (ranging from 0 to 50 $\mathrm{nM}$ ) for both sequences diluted in the hybridization buffer. By applying these conditions, we achieved limits of detection of 387 and 438 pM for Fas57 and Fas567 isoforms, respectively. Such detection limits prove the excellent sensitivity of our methodology for the direct and label-free assay of nucleic acids, which is usually in the nM-pM range ${ }^{31}$.

SPR quantitative data was obtained by interpolation of the SPR response in the calibration curve equation specific for each isoform. Figure 4C shows the quantification of the two isoforms under each condition. Quantitative data obtained from non-fragmented samples $\left(\mathrm{SPR}^{0}\right)$ were $24.6 / 5.5 \mathrm{nM}$ for 
Fas567/Fas57. In contrast, the quantities detected increased by a factor of ten after the application of either fragmentation protocol $\left(262 / 12 \mathrm{nM}\right.$ for $\mathrm{SPR}^{\Delta}$ and 295/13.1 nM for $\left.\mathrm{SPR}^{\mathrm{H}}\right)$.

To validate the results and the methodology introduced in this work for site-specific mRNA cleavage, we carried out parallel analysis of HeLa RNA samples with RT-qPCR. Table 3 shows the comparison between the quantification performed by RTqPCR and the three different approaches carried out using the SPR biosensor. According to RT-qPCR data (365/20.3 nM), SPR results from non-fragmented samples heavily underestimated RNA abundance (accuracy lower than 30\%), confirming the need for a fragmentation process.

RNase H cleavage results showed $81 \%$ accuracy for Fas567 and $65 \%$ for Fas57 compared to RT-qPCR, whereas alkaline hydrolysis showed accuracies of $65 \%$ for Fas567 and 59\% for Fas 57. Thus, RNase H outperformed alkaline hydrolysis up to $20 \%$ for the detection of the different isoforms, leveraging this approach for a more accurate detection of AS events. The slight underestimation of isoform concentrations by $\mathrm{SPR}^{\mathrm{H}}$ compared to the results from the RT-qPCR could be due to limitations in the sensitivity of the SPR biosensor, since analyses are performed in an amplification-free and label-free scheme.

Finally, to further confirm the advantages of the RNase $\mathrm{H}$ fragmentation, we applied the procedure to quantify Fas (567/57) isoform ratios in a highly accurate and selective manner (Figure 4D). Isoform ratio is a key feature to predict the aberrant regulation of AS events. Therefore, it is the most important parameter in order to test the efficiency of AS event analysis. As can be appreciated in the figure, the ratio between isoforms is very different in the absence of fragmentation. According to $\mathrm{SPR}^{\mathrm{H}}$ results, Fas567 isoform had increased expression compared to Fas57 isoform, leading to an isoform ratio $(567 / 57)$ of $22.0 \pm 0.7$. These results show a good correlation with the RT-qPCR $(567 / 57=17.9 \pm 0.4)$. Also, in order to test the agreement between the two techniques, different RNA samples presenting different isoform ratios were analysed and compared by SPR ${ }^{\mathrm{H}}$ and RT-qPCR. To induce different isoform ratios, HeLa cells were transfected with a minigene expressing $F a$ s genomic sequences. Then, total RNA was purified either alone or co-transfected with a vector expressing the Polypyrimidine-Tract-Binding protein (PTB). PTB induces skipping of Fas exon 6 acting through an exonic silencer sequence ${ }^{29}$. While expression of the minigene alone leads to preferential accumulation of exon 6-including transcripts, co-expression of Fas567 minigene with PTB leads to higher accumulation of transcripts that skip exon $6^{28}$. Each condition was analysed by $\mathrm{SPR}^{\mathrm{H}}$ and RT-qPCR and isoform ratios were calculated. All samples tested (6/6) were found within the limits of agreement (Figure S3). In fact, the agreement between SPR measurements and RT-PCR is higher for RNase $\mathrm{H}$ digestion compared to alkaline hydrolysis performed in our previous work by a $20 \%$ (83\% agreement), confirming that RNase $\mathrm{H}$ cleavage is a more accurate approach for the analysis of AS isoforms in an amplification-free and label-free detection system.
Table 3. RT-qPCR and SPR comparison of the isoform content analysis from HeLa cells endogenous expression. Isoform concentration in $\mathrm{nM}$ for SPR was calculated through the calibration curves obtained for each receptor according to their $\Delta \mathrm{R}(\%)$.

\begin{tabular}{cccc}
\hline \multirow{2}{*}{$\begin{array}{c}\text { Fas } \\
\text { isoform }\end{array}$} & \multicolumn{2}{c}{ Concentration (nM) } \\
\cline { 3 - 4 } & RT-qPCR* & SPR* $^{*}$ & Fragmentation \\
\hline \multirow{2}{*}{$\mathbf{5 6 7}$} & $365 \pm 17^{\mathrm{a}}$ & $24.6 \pm 0.40$ & No fragmentation \\
\cline { 3 - 4 } & & $262 \pm 27$ & Alkaline hydrolisis \\
\cline { 3 - 4 } & & $295 \pm 14$ & RNaseH cleavage \\
\cline { 3 - 4 } $\mathbf{5 7}$ & $20.3 \pm 0.4^{\mathrm{a}}$ & $12.0 \pm 0.8$ & No fragmentation \\
\cline { 3 - 4 } & & $13.1 \pm 0.3$ & Rlkaline hydrolisis \\
\hline
\end{tabular}

*Mean \pm SD of endogenous concentration of HeLa cell's total RNA calculated from 2 different samples in each experiment.

\section{CONCLUSION}

Alternative splicing can contribute to the development and progression of human malignancies and it has been highlighted as a hallmark of cancer. The use of alternatively spliced isoforms as biomarkers will likely increase in the near future. Evaluating the impact of AS variants in cancer could be adopted as an integral part of clinical diagnosis. In addition, modulation of isoform ratios poses great promise in future precision medicine for personalized therapies.

Despite the significant advances in optical biosensors and their potential application for rapid diagnostics tests, there is a lack of applications related to long RNA sequences, such as mRNA isoforms, due to the difficult access of these sequences to their specific DNA-probes in the biosensor bioreceptor monolayer. We show that this biosensing approach can be greatly enhanced by previous sample pre-processing steps to generate targets with the desired length that will not display the limitations associated to long RNAs.

We have adopted a methodology for the site-specific cleavage of mRNA sequences in order to obtain fragments with a precise sequence and length of interest. It has been employed for the fast and direct monitoring of alternative splicing events in real-time using an SPR biosensor. Our methodology involves the design of specific DNA oligonucleotides that hybridize the flanking segments of the RNA fragment of interest, promoting their degradation by RNase $\mathrm{H}$ activity. This approach permits the generation of mRNA fragments with the appropriate length with high selectivity, ensuring their accurate quantification via optical biosensors.

The feasibility of the methodology was tested in complex RNA mixtures purified from HeLa cells expressing endogenous Fas gene mRNA transcripts. The results strongly correlated with RT-qPCR data, with a detection accuracy up to $81 \%$. In addition, they showed higher correlation between the RNase H cleavage and RT-qPCR compared with the conventional fragmentation process based on alkaline hydrolysis by a $20 \%$. These results confirm that site-specific RNA fragmentation reduces errors in isoform quantification, probably due to a lower loss of sample input than when applying random fragmentation. A slight underestimation of the isoform concentrations was observed in the SPR analysis, which could be probably due to limitations in the sensor sensitivity. One solution could be the implementation of this methodology in other la- 
bel-free optical biosensors. For example, bimodal waveguide interferometers (BiMW) have demonstrated to outperform SPR sensitivities up to three orders of magnitude in the detection of AS events ${ }^{32}$.

Our methodology, along with the label-free detection and the minimized sample manipulation, provides an advance in the real-time monitoring of AS events. In our view, these results open the door for the routine use of alternatively spliced isoforms as biomarkers for diagnosis and patient follow-ups during therapy. In addition, it tackles the challenges encountered in the analysis of long RNA sequences with label-free optical biosensors, enabling to exploit the potential of these biosensors for highly sensitive, selective, fast and userfriendly genomic and epigenomic analyses. Moreover, it can be implemented in more integrated and sensitive platforms that allow for multiplexed analysis of multiple AS events ${ }^{32}$. This work provides a significant step forward for the development of sensitive diagnostic tools based on nucleic acid biomarker detection for cancer research.

\section{ASSOCIATED CONTENT}

\section{Supporting Information}

Additional information as noted in text. The Supporting Information is available free of charge on the ACS Publications website.

\section{AUTHOR INFORMATION}

\section{Corresponding Author}

*E-mail: cesar.sanchez.huertas@ rmit.edu.au

\section{Author Contributions}

CSH, JV and LML devised the idea and designed the experiments. CSH performed the SPR experiments. SB performed the RT-qPCR validation experiments and provided the HeLa cell samples. CSH, SB, MS, JV and LML analyzed the data. The manuscript was written through contributions of all authors. / All authors have given approval to the final version of the manuscript.

\section{ACKNOWLEDGMENT}

We acknowledge the financial support from PreDICT project (Programa estatal de investigación, desarrollo e innovación orientada a los Retos de la Sociedad, TEC2016-78515-R). SPR studies were performed at the ICTS NANBIOSIS, especifically at the Biodeposition and Biodetection Unit (http://www.nanbiosis.es/portfolio/u4-biodeposition-and-

biodetection-unit/). ICN2 is the recipient of Grant SEV-20170706 from the "Severo Ochoa Centers of Excellence" Program of Spanish Ministry of Science. Work in the JV laboratory is supported by the European Research Council (ERC AdvG 670146), AGAUR, Spanish Ministry of Economy and Competitiveness (BFU 2014-005153 and BFU 2017 89308-P) and the Centre of Excellence Severo Ochoa.

CSH is a recipient of an RMIT Vice Chancellor's Postdoctoral Fellowship.

\section{REFERENCES}

(1) Scotti, M. M.; Swanson, M. S. RNA Mis-Splicing in Disease Nat. Rev. Genet. 2016, 17 (1), 19-32. https://doi.org/10.1038/nrg.2015.3.
Oltean, S.; Bates, D. O. Hallmarks of Alternative Splicing in Cancer. Oncogene 2013, No. November, 1-8. https://doi.org/10.1038/onc.2013.533.

Ge, Y.; Weygant, N.; Qu, D.; May, R.; Berry, W. L.; Yao, J.; Chandrakesan, P.; Zheng, W.; Zhao, L.; Zhao, K. L.; et al. Alternative Splice Variants of DCLK1 Mark Cancer Stem Cells, Promote Self-Renewal and Drug-Resistance, and Can Be Targeted to Inhibit Tumorigenesis in Kidney Cancer. Int. J. Cancer 2018, $143 \quad$ (5), $1162-1175$. https://doi.org/10.1002/ijc.31400.

Park, E.; Pan, Z.; Zhang, Z.; Lin, L.; Xing, Y. The Expanding Landscape of Alternative Splicing Variation in Human Populations. Am. J. Hum. Genet. 2018, 102 (1), 11-26. https://doi.org/10.1016/j.ajhg.2017.11.002.

Wang, B.-D.; Ceniccola, K.; Hwang, S.; Andrawis, R.; Horvath, A.; Freedman, J. A.; Olender, J.; Knapp, S.; Ching, T.; Garmire, L.; et al. Alternative Splicing Promotes Tumour Aggressiveness and Drug Resistance in African American Prostate Cancer. Nat. Commun. 2017, 8, 15921. https://doi.org/10.1038/ncomms15921.

Zhao, S. Alternative Splicing, RNA-Seq and Drug Discovery. Drug Discov. Today 2019. https://doi.org/10.1016/j.drudis.2019.03.030.

Alix-Panabières, C.; Pantel, K. Clinical Applications of Circulating Tumor Cells and Circulating Tumor DNA as Liquid Biopsy. Cancer Discov. 2016, 6 (5), 479-491. https://doi.org/10.1158/2159-8290.CD-15-1483.

Suñé-Pou, M.; Prieto-Sánchez, S.; Boyero-Corral, S.; MorenoCastro, C.; El Yousfi, Y.; Suñé-Negre, J. M.; HernándezMunain, C.; Suñé, C. Targeting Splicing in the Treatment of Human Disease. Genes (Basel). 2017, 8 (3). https://doi.org/10.3390/genes8030087.

Martinez-Montiel, N.; Rosas-Murrieta, N. H.; Anaya Ruiz, M.; Monjaraz-Guzman, E.; Martinez-Contreras, R. Alternative Splicing as a Target for Cancer Treatment. Int. J. Mol. Sci. 2018, 19 (2). https://doi.org/10.3390/ijms19020545.

Harvey, S. E.; Cheng, C. Methods for Characterization of Alternative RNA Splicing. Methods Mol. Biol. 2016, 1402, 229241. https://doi.org/10.1007/978-1-4939-3378-5_18.

Lee, C.; Roy, M. Analysis of Alternative Splicing with Microarrays: Successes and Challenges. Genome Biol. 2004, 5 (7), 231. https://doi.org/10.1186/gb-2004-5-7-231.

Castle, J. C.; Zhang, C.; Shah, J. K.; Kulkarni, A. V; Kalsotra, A.; Cooper, T. A.; Johnson, J. M. Expression of 24,426 Human Alternative Splicing Events and Predicted Cis Regulation in 48 Tissues and Cell Lines. Nat. Genet. 2008, 40 (12), 1416-1425. https://doi.org/10.1038/ng.264.

Carrascosa, L. G.; Huertas, C. S.; Lechuga, L. M. Prospects of Optical Biosensors for Emerging Label-Free RNA Analysis. TrAC - Trends in Analytical Chemistry. Elsevier June 1, 2016, pp 177-189.

Soler, M.; Huertas, C. S.; Lechuga, L. M. Label-Free Plasmonic Biosensors for Point-of-Care Diagnostics: A Review. Expert Rev. Mol. Diagn. 2018. https://doi.org/10.1080/14737159.2019.1554435.

LeProust, E. M.; Peck, B. J.; Spirin, K.; McCuen, H. B.; Moore, B.; Namsaraev, E.; Caruthers, M. H. Synthesis of High-Quality Libraries of Long (150mer) Oligonucleotides by a Novel Depurination Controlled Process. Nucleic Acids Res. 2010, 38 (8), 2522-2540. https://doi.org/10.1093/nar/gkq163.

Huertas, C. S.; Carrascosa, L. G.; Bonnal, S.; Valcárcel, J.; Lechuga, L. M. Quantitative Evaluation of Alternatively Spliced MRNA Isoforms by Label-Free Real-Time Plasmonic Sensing. Biosens. Bioelectron. 2016, 78, 118-125.

Sasi, M. S.; Mlitan, A. M.; Alkherraz, A. M. Comparing Spontaneous Hydrolysis Rates of Activated Models of DNA and RNA. 2014.

Cerritelli, S. M.; Crouch, R. J. Ribonuclease H: The Enzymes in Eukaryotes. FEBS J. 2009, 276 (6), 1494-1505. https://doi.org/10.1111/j.1742-4658.2009.06908.x.

Uyeno, Y.; Sekiguchi, Y.; Sunaga, A.; Yoshida, H.; Kamagata, Y. Sequence-Specific Cleavage of Small-Subunit (SSU) RRNA with Oligonucleotides and RNase H: A Rapid and Simple 
Approach to SSU RRNA-Based Quantitative Detection of Microorganisms. Appl. Environ. Microbiol. 2004, 70 (6), 36503663. https://doi.org/10.1128/AEM.70.6.3650-3663.2004.

(20) Krammer, P. H. CD95's Deadly Mission in the Immune System. Nature $\quad 2000, \quad 407 \quad$ (6805), $789-795$ https://doi.org/10.1038/35037728.

(21) Cheng, J.; Zhou, T.; Liu, C.; Shapiro, J. P.; Brauer, M. J.; Kiefer, M. C.; Barr, P. J.; Mountz, J. D. Protection from FasMediated Apoptosis by a Soluble Form of the Fas Molecule. Science 1994, 263 (5154), 1759-1762.

(22) Cascino, I.; Fiucci, G.; Papoff, G.; Ruberti, G. Three Functional Soluble Forms of the Human Apoptosis-Inducing Fas Molecule Are Produced by Alternative Splicing. J. Immunol. 1995, 154 (6), 2706-2713.

(23) Owen-Schaub, L.; Chan, H.; Cusack, J. C.; Roth, J.; Hill, L. L. Fas and Fas Ligand Interactions in Malignant Disease. Int. J. Oncol. 2000, 17 (1), 5-12.

(24) Owen-Schaub, L. B.; van Golen, K. L.; Hill, L. L.; Price, J. E. Fas and Fas Ligand Interactions Suppress Melanoma Lung Metastasis. J. Exp. Med. 1998, 188 (9), 1717-1723. https://doi.org/10.1084/jem.188.9.1717.

(25) Opdahl, A.; Petrovykh, D. Y.; Kimura-Suda, H.; Tarlov, M. J.; Whitman, L. J. Independent Control of Grafting Density and Conformation of Single-Stranded DNA Brushes. Proc. Natl. Acad. Sci. 2007. https://doi.org/10.1073/pnas.0608568103.

(26) Carrascosa, L. G.; Calle, A.; Lechuga, L. M. Label-Free Detection of DNA Mutations by SPR: Application to the Early Detection of Inherited Breast Cancer. Anal. Bioanal. Chem. 2009. https://doi.org/10.1007/s00216-008-2555-1.

(27) Huertas, C. S.; Aviñó, A.; Kurachi, C.; Piqué, A.; Sandoval, J.; Eritja, R.; Esteller, M.; Lechuga, L. M. Label-Free DNAMethylation Detection by Direct Ds-DNA Fragment Screening Using Poly-Purine Hairpins. Biosens. Bioelectron. 2018. https://doi.org/10.1016/j.bios.2018.08.027.

(28) Förch, P.; Puig, O.; Kedersha, N.; Martínez, C.; Granneman, S.; Séraphin, B.; Anderson, P.; Valcárcel, J. The ApoptosisPromoting Factor TIA-1 Is a Regulator of Alternative PreMRNA Splicing. Mol. Cell 2000, 6 (5), 1089-1098.

(29) Izquierdo, J. M.; Majós, N.; Bonnal, S.; Martínez, C.; Castelo, R.; Guigó, R.; Bilbao, D.; Valcárcel, J. Regulation of Fas Alternative Splicing by Antagonistic Effects of TIA-1 and PTB on Exon Definition. Mol. Cell 2005, 19 (4), 475-484. https://doi.org/10.1016/j.molcel.2005.06.015.

(30) Joshi, G. K.; Deitz-Mcelyea, S.; Johnson, M.; Mali, S.; Korc, M.; Sardar, R. Highly Specific Plasmonic Biosensors for Ultrasensitive MicroRNA Detection in Plasma from Pancreatic Cancer Patients. 2014. https://doi.org/10.1021/n1503220s.

(31) Šípová, H.; Homola, J. Surface Plasmon Resonance Sensing of Nucleic Acids: A Review. Analytica Chimica Acta. 2013, pp 923. https://doi.org/10.1016/j.aca.2012.12.040.

(32) Huertas, C. S.; Domínguez-Zotes, S.; Lechuga, L. M. Analysis of Alternative Splicing Events for Cancer Diagnosis Using a Multiplexing Nanophotonic Biosensor. Sci. Rep. 2017, 7, 41368. https://doi.org/10.1038/srep41368. 


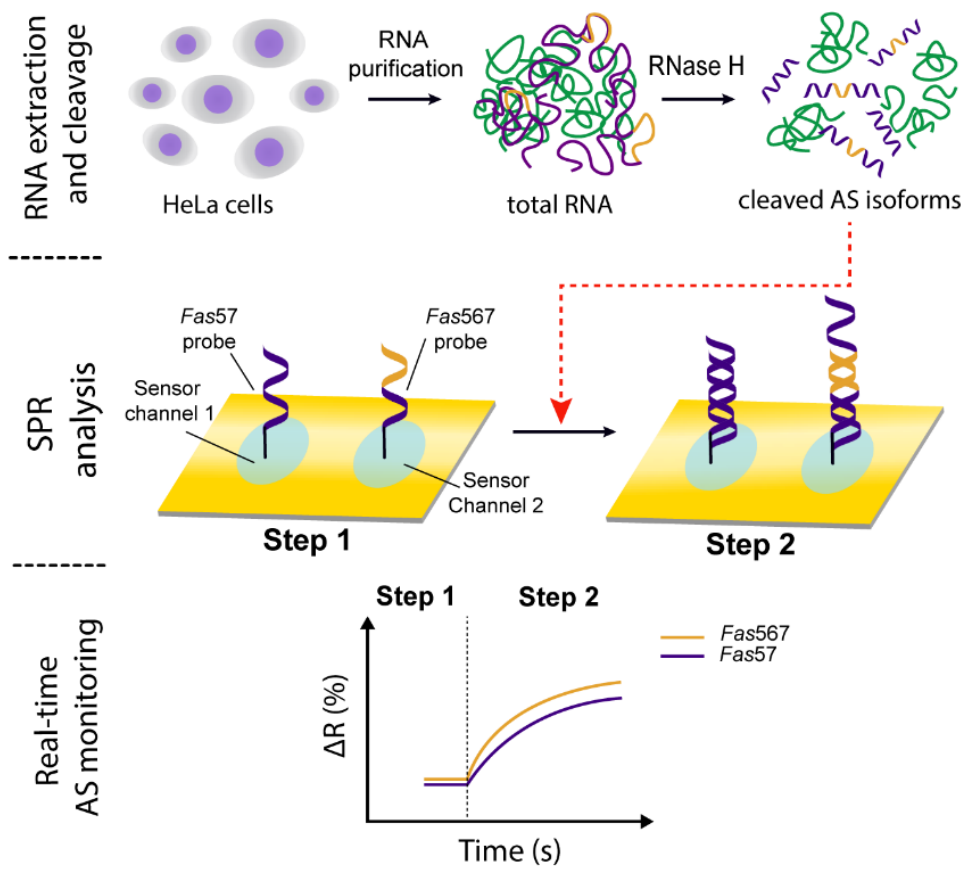

